Iżecka Joanna. Hydrotherapy in nervous system diseases. Journal of Education, Health and Sport. 2019;9(1):55-60. eISNN 23918306. DOI http://dx.doi.org/10.5281/zenodo.2535943

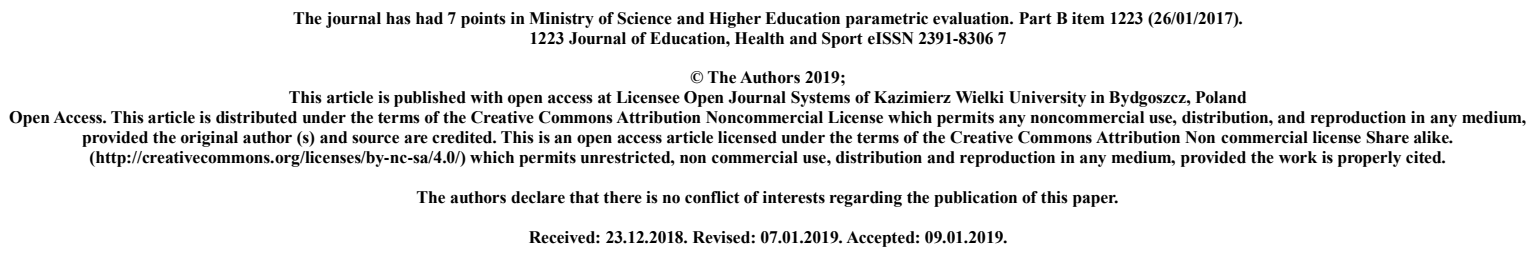

\title{
Hydrotherapy in nervous system diseases
}

\author{
Joanna Iłżecka \\ Independent Neurological Rehabilitation Unit, Medical University of Lublin
}

\section{SUMMARY}

Hydrotherapy is one method used in the rehabilitation treatment of various diseases, including diseases of the nervous system with a positive result. It is used in many exercise programs. It has been shown that hydrotherapy reduces the clinical symptoms of the nervous system diseases, reduces pain and improves quality of life. The mechanism of action of hydrotherapy is omnidirectional; among other things, it has a positive effect on segmental spinal mechanisms, induces muscle relaxation, affects the activity of the autonomic nervous system. The aim of the work was a review of the literature on hydrotherapy in nervous system diseases.

Keywords: hydrotherapy, nervous system diseases, rehabilitation

\section{INTRODUCTION AND PURPOSE OF WORK}

Hydrotherapy - aquatic therapy - is a form of rehabilitation used in many exercise programs. Water acts as a unique medium allowing for weight-bearing exercise without stressing the joints, movement and stability drills without the fear of falling, and multidirectional resistance training without the need for free weights or bands. Hydrotherapy is performed in a warm (above $30^{\circ} \mathrm{C}$ ), shallow pool and may involve a variety of exercise including aerobic, stretching/range of motion, resistance, and stability training [1,2]. A pool therapy program offers special populations the opportunity to complete movement tasks in the water that are no longer feasible on land [1]. It was observed that aquatherapy can improve a quality of life and mobility-related activities of daily living [3].

Hydrotherapy is frequently applied to patients with painful neurological or musculoskeletal alterations, because the heat and floatability of the water can block nociceptors by acting on thermal receptors and mechanoreceptors and exert a positive effect on spinal segmental mechanisms. Warm water can also increase the blood flow, helping to dissipate allogeneic chemicals and enhance muscle relaxation. The hydrostatic effect of water 
can alleviate pain by reducing peripheral edema and sympathetic nervous system activity [47].

The aim of the work was a review of the literature on hydrotherapy in nervous system diseases.

\section{HYDROTHERAPY IN NERVOUS SYSTEM DISEASES - REVIEW OF THE LITERATURE}

Marinho-Buzelli et al. [8] investigated evidence on the effects of hydrotherapy on mobility in patients with different neurological diseases. Two randomized controlled trials (30 patients with stroke in the hydrotherapy groups), three non-randomized studies and three before-and-after studies showed "fair" evidence that aquatic therapy can increase dynamic balance in patients with some neurological disorders. One randomized controlled trial (7 patients with stroke in the hydrotherapy group) and two before-and-after tests (20 patients with multiple sclerosis) demonstrated that hydrotherapy improved of gait speed in participants. According to authors the studies showed "fair" evidence supporting the use of hydrotherapy to improve dynamic balance and gait speed in patients with certain neurological disorders.

Eyvaz et al. [9] determined whether the water based exercise (WBE) program applied in combination with the land-based exercises (LBE) compared to LBE alone contributes to the stroke patients' motor functions, walking, balance functions and quality of life. In total, 60 patients participated in this study. WBE therapy (3/week) + LBE (2/week) was applied to the patients in the study group for six weeks. LBE was applied to the control group 5/week for six weeks. The results showed significant improvements in all of the investigated parameters (except SF - 36 pain parameter) in both groups. The authors concluded that applying WBE together with the LBE (except SF-36 vitality sub-parameter) in patients with hemiplegia did not make any additional contribution to the application of LBE alone.

Pinto et al. [10] reviewed the effects of hydrotherapy on balance, motor status, functional mobility, and quality of life in patients with Parkinson's disease (PD). A total of 484 participants were included to the study. The meta-analysis showed a moderate-quality of evidence for positive effects of hydrotherapy combined or not with land-based therapy on balance and functional mobility. The authors found a low quality of evidence for no effects of hydrotherapy combined or not with land-based therapy on quality of life and on motor status. Pinto et al. concluded that hydrotherapy combined or not with other therapies may improve balance and functional mobility in patients with PD when compared to land-based therapy alone or usual care.

Ayán et al. [11] investigated the effects of two different water-based exercise training programs on functional mobility, motor symptoms, and quality of life (QOL) in patients with PD. Participants were assigned to a low-intensity water exercise program (group 1) or a muscular resistance water exercise intervention (group 2). Group sessions were held for 12 weeks, twice a week for 60 minutes per session. Results showed a significant improvement in QOL of PD patients. Only participants in group 2 showed a significant change in functional mobility and motor symptoms. The authors concluded that waterbased exercise training programs in PD are helpful therapy and muscular resistance programs of this kind are efficient in improving functional mobility, motor symptoms, and QOL of PD patients.

Hind et al. [12] assessed aquatic therapy for boys with Duchenne muscular dystrophy. Ambulant boys aged 7-16 years established on steroids, with North Star Ambulatory Assessment (NSAA) score $\geq 8$, who were able to complete a 10 -m walk test without aids or 
assistance, were randomly allocated to 6 months of either optimized land-based exercises 4 to 6 days/week, or the same 4 days/week plus aquatic therapy 2 days/week. The main outcome measures included feasibility of recruiting 40 participants in 6 months from six UK centers, clinical outcomes including NSAA, independent assessment of treatment optimization, participant/therapist views on acceptability of intervention and research protocols, value of information analysis and cost-impact analysis. Over 6 months, 348 boys were screened. The mean change in NSAA at 6 months was -5.5 in the control arm and -2.8 in the aquatic therapy arm. Harms included fatigue in two boys, pain in one. The authors concluded that the study can help in the optimization of aquatic therapy service provision and the design of future research.

Kargarfard et al [13] examined the effectiveness of aquatic exercise training on fatigue and health-related quality of life (HRQOL) in women with multiple sclerosis (MS).The intervention consisted of 8 weeks supervised aquatic exercise in a swimming pool (3 times a week, each session lasting $60 \mathrm{~min})$. Twenty one patients took part in the study (10 - exercise group and 11 control group). There was no significant difference between the 2 groups at the baseline. Patients in the aquatic exercise group showed significant improvements in fatigue and HRQOL after 4 and 8 weeks compared with the control group. The authors concluded that aquatic exercise training can effectively improve fatigue and HRQOL of patients with MS and should be considered in the management of this relatively common public health problem.

Salem et al. [14] determined the feasibility of providing a community based aquatic exercise programme and examined the effects of a group aquatic programme in individuals with MS. Eleven subjects with MS participated in a 5-week community based aquatic exercise programme. Aquatic exercises were held twice weekly for 60 minutes and included aerobic exercises, strength training, flexibility exercises, balance training and walking activities. The 10-Metre Walk test, the Berg Balance Scale (BBS), the 'Timed Up and Go' (TUG) test, grip strength and the Modified Fatigue Impact Scale were used to assess motor function. Analysis of the scores demonstrated improved gait speed, BBS, TUG test and grip strength. All participants reported that they enjoyed the programme, and they had improved after the training. The authors concluded that a community-based aquatic exercise programme is feasible and resulted in improvement in motor functions of individuals with MS.

Peterson [15] described the examination, intervention, and outcome of a patient with MS who participated in rehabilitation program that included hydrotherapy with a pool temperature of $94^{\circ} \mathrm{F}$. The patient did not experience heat sensitivity or fatigue throughout the program. Result showed the improvement manual muscle test grades and mobility of patient. The author concluded that this patient's participation in aquatic therapy, in conjunction with land-based interventions, may have been associated with the improvement in functional abilities.

Broach et al. $[16,17]$ examined aquatic therapy in patients with MS and observed an improvement in motor function and strength, and reported that these programs were enjoyable.

Castro-S' anchez et al. [18] investigated the effectiveness of an Ai-Chi aquatic exercise program against pain and other symptoms in MS patients. In this randomized controlled trial, 73 MS patients were assigned to an investigated or control group for a 20-week treatment program. The investigated group underwent 40 sessions of Ai-Chi exercise in swimming pool and the control group 40 sessions of abdominal breathing and contraction-relaxation exercises in therapy room. The authors assessed pain, disability, spasm, depression, fatigue, and autonomy, before the therapy and immediately and at 4 and 10 weeks after the last treatment session. The investigated group showed a significant and clinically relevant decrease in pain intensity, with an immediate posttreatment reduction in median visual analogue scale scores of $50 \%$ that was maintained for up to 10 weeks. Significant improvements were also observed in spasm, fatigue, disability, and autonomy. The authors concluded that an Ai-Chi aquatic 
exercise program improves pain, spasms, disability, fatigue, depression, and autonomy in MS patients.

Piskorz et al. [19] examined 41 patients with type 2 diabetes and neuropathic pain. Participants were randomly divided into group A (24 patients - massage whirlpool), group B (17 patients - electro-water bath). Patients in group A and B were used treatments lasting 20 minutes - a series of 10 hydrotherapy treatments at intervals of 2-3 days. The authors concluded that there were statistically significant changes in the assessment of pain measured before and after hydrotherapy in both groups, and the group as a whole. Prior treatments pain values were significantly higher than after therapy. In group B, greater analgesic efficacy compared with A was observed. According to authors the long-term effect of combination therapy in the form of drug therapy and hydrotherapy is unknown and requires further research.

Sujan et al. [20] assessed influence of hydrotherapy on clinical and cardiac autonomic function in migraine patients. Forty chronic migraine patients were randomized to receive hydrotherapy plus conventional pharmacological care or conventional medication only. Hydrotherapy group received treatment with hot arm and foot bath $\left(103^{\circ} \mathrm{F}\right.$ to $\left.110^{\circ} \mathrm{F}\right)$ and ice massage to head daily for $20 \mathrm{~min}$ for 45 days. Patients were assessed using headache impact test (HIT), visual analog scale for pain and cardiac autonomic function by heart rate variability (HRV) before and after intervention period. There was a significant decrease in HIT score, frequency, and intensity of headaches following treatment in both the groups. However, it was more evident in add on hydrotherapy group compared to pharmacological treatment alone group. There was also significant improvement in the HRV parameters. In particular, there was a significant decrease in heart rate increase in high frequency and decrease in low frequency/HF ratio in add on hydrotherapy group. The authors concluded that add on hydrotherapy enhanced the vagal tone in addition to reducing the frequency and intensity of headaches in migraine patients. According to authors mechanism by which hydrotherapy brings positive effects remains speculative. Sensory stimuli of hydrotherapy may dominate the painful stimuli by activation of large diameter myelinated fibers. This may lead to loss of gate to small nociceptive impulses. Both hypo and hyperthermia are known to exert strong physiological and hemodynamic effects on the body. Studies have shown that warm water exposure decreases sympathetic power and increase vagal tone. The vasogenic inflammation and constriction that are hallmarks of migraine may be modulated by thermal applications resulting in clinical improvement [20-23].

Johnson [24] described aquatic therapy for patient with amyotrophic lateral sclerosis (ALS). Ellapen et al. [25] discussed the benefits of hydrotherapy to spinal cord injuries concerning underwater gait-kinematics, thermoregulatory and cardiovascular responses and spasticity. The authors concluded that hydrotherapy improves spinal cord injury underwater gait-kinematics, cardiorespiratory and thermoregulatory responses and reduces spasticity.

\section{CONCLUSION}

Hydrotherapy is one of the forms of rehabilitation of patients with diseases of the nervous system. Data from the literature indicate that significantly improves the clinical status of patients and their quality of life.

\section{REFERENCES}

1. Bartels EM, Lund H, Hagen KB, Dagfinrud H, Christensen R, Danneskiold-Samsøe B. Aquatic exercise for the treatment of knee and hip osteoarthritis. Cochrane Database Syst Rev. 2007; 4: CD005523. 
2. Plecash AR, Leavitt BR. Aquatherapy for neurodegenerative disorders. J Huntingtons Dis. 2014; 3(1): 5-11.

3. Sato D, Kaneda K, Wakabayashi H, Nomura T. Comparison two-year effects of onceweekly and twice weekly water exercise on health-related quality of life of community-dwelling frail elderly people at a day-service facility. Disabil Rehabil. 2009; 31(2): 84-93.

4. Kamioka H, Tsutani K, Okuizumi H, Mutoh Y, Ohta M, Handa S, et al. Effectiveness of aquatic exercise and balneotherapy: a summary of systematic reviews based on randomized controlled trials of water immersion therapies. J Epidemiol. 2010; 20(1): 2-12.

5. Hall J, Swinkels A, Briddon J, McCabe CS. Does aquatic exercise relieve pain in adults with neurologic or musculoskeletal disease? a systematic review and metaanalysis of randomized controlled trials. Arch Phys Med Rehabil. 2008; 89(5): 873883.

6. Bender T, Karagülle Z, Bálint GP, Gutenbrunner C, Bálint $\quad$ PV, Sukenik S. Hydrotherapy, balneotherapy, and spa treatment in pain management. Rheumatol Int. 2005; 25(3): 220-224.

7. Gabrielsen A, Videbaek R, Johansen LB, Warberg J, Christensen NJ, Pump B, et al. Forearm vascular and neuroendocrine responses to graded water immersion in humans. Acta Physiol Scand. 2000; 169(2): 87-94.

8. Marinho-Buzelli AR, Bonnyman AM, Verrier MC. The effects of aquatic therapy on mobility of individuals with neurological diseases: a systematic review. Clin Rehabil. 2015; 29(8): 741-751.

9. Eyvaz N, Dundar U, Yesil H. Effects of water-based and land-based exercises on walking and balance functions of patients with hemiplegia. NeuroRehabilitation. 2018; 43(2): 237-246.

10. Pinto C, Salazar AP, Marchese RR, Stein C, Pagnussat AS. Is hydrotherapy effective to improve balance, functional mobility, motor status, and quality of life in subjects with Parkinson's disease? A systematic review and meta-analysis. PMR. 2018 Sep 25. pii: S1934-1482(18)30931-6. doi: 10.1016/j.pmrj.2018.09.031.

11. Ayán C, Cancela J. Feasibility of 2 different water-based exercise training programs in patients with Parkinson's disease: a pilot study. Arch Phys Med Rehabil. 2012; 93(10): 1709-1714.

12. Hind D, Parkin J, Whitworth V, Rex S, Young T, Hampson L, et al. Aquatic therapy for boys with Duchenne muscular dystrophy (DMD): an external pilot randomised controlled trial. Pilot Feasibility Stud. 2017; 3: 16.

13. Kargarfard M, Etemadifar M, Baker P, Mehrabi M, Hayatbakhsh R. Effect of aquatic exercise training on fatigue and health-related quality of life in patients with multiple sclerosis. Arch Phys Med Rehabil. 2012; 93(10): 1701-1708.

14. Salem Y, Scott AH, Karpatkin H, Concert G, Haller L, Kaminsky E, et al. Community-based group aquatic programme for individuals with multiple sclerosis: a pilot study. Disabil Rehabil. 2011; 33(9): 720-728.

15. Peterson C. Exercise in 94 degrees $\mathrm{F}$ water for a patient with multiple sclerosis. Phys Ther. 2001; 81(4): 1049-1058.

16. Broach E, Dattilo J. Effects of aquatic therapy on adults with multiple sclerosis. Ther Recreation J. 2001; 35: 141-154.

17. Broach E, Dattilo J, McKenney A. Effects of aquatic therapy on perceived fun or enjoyment experiences of participants with multiple sclerosis. Ther Recreation J. 2007; 41: 179-200. 
18. Castro-S'anchez AM, Matar'an-Pe narrocha GA, Lara-Palomo I, SaavedraHern'andez M, Arroyo-Morales M, Moreno-Lorenzo C. Hydrotherapy for the treatment of pain in people with multiple sclerosis: a randomized controlled trial. Evid Based Complement Alternat Med. 2012; 2012: 473963.

19. Piskorz J, Wójcik G, Pisz K, Nalepa D, Skalska-Izdebska R, Bulikowski W. Hydrotherapy as a treatment method of supporting of neuropathic pain in diabetes preliminary reports. Acta Balneol. 2015; 67, 4 (142): 271-276.

20. Sujan MU, Rao MR, Kisan R, Abhishekh HA, Nalini A, Raju TR, et al. Influence of hydrotherapy on clinical and cardiac autonomic function in migraine patie nts. J Neurosci Rural Pract. 2016; 7(1): 109-113.

21. Mooventhan A, Nivethitha L. Scientific evidence-based effects of hydrotherapy on various systems of the body. N Am J Med Sci. 2014; 6: 199-209.

22. Perini R, Veicsteinas A. Heart rate variability and autonomic activity at rest and during exercise in various physiological conditions. Eur J Appl Physiol. 2003; 90: 317-325.

23. Nishimura M, Onodera S. Effects of water temperature on cardiac autonomic nervous system modulation during supine floating. J Gravit Physiol. 2001; 8: 65-66.

24. Johnson CR. Aquatic therapy for an ALS patient. Am J Occup Ther. 1988; 42(2): 115120.

25. Ellapen TJ, Hammill HV, Swanepoel M, Strydom GL. The benefits of hydrotherapy to patients with spinal cord injuries. Afr J Disabil. 2018; 7(0): 450. doi: 10.4102/ajod.v7i0.450. 